\title{
Noninvasive analysis of metabolic changes following nutrient input into diverse fish species, as investigated by metabolic and microbial profiling approaches
}

An NMR-based metabolomic approach in aquatic ecosystems is valuable for studying the environmental effects of pharmaceuticals and other chemicals on fish. This technique has also contributed to new information in numerous research areas, such as basic physiology and development, disease, and water pollution. We evaluated the microbial diversity in various fish species collected from Japan's coastal waters using next-generation sequencing, followed by evaluation of the effects of feed type on co-metabolic modulations in fish-microbial symbiotic ecosystems in laboratory-scale experiments. Intestinal bacteria of fish in their natural environment were characterized (using 16S rRNA genes) for trophic level using pyrosequencing and noninvasive sampling procedures developed to study the metabolism of intestinal symbiotic ecosystems in fish reared in their environment. Metabolites in feces were compared, and intestinal contents and feed were annotated based on HSQC and TOCSY using SpinAssign and network analysis. Feces were characterized by species and varied greatly depending on the feeding types. In addition, feces samples demonstrated a response to changes in the time series of feeding. The potential of this approach as a non-invasive inspection technique in aquaculture is suggested. 
1 Noninvasive analysis of metabolic changes following nutrient input into diverse fish species,

2 as investigated by metabolic and microbial profiling approaches

3 Taiga Asakura ${ }^{1,2}$, Kenji Sakata ${ }^{1}$, Seiji Yoshida ${ }^{1,2}$, Yasuhiro Date ${ }^{1,2}$ and Jun Kikuchi ${ }^{1,2,3,4, *}$

4 'RIKEN Center for Sustainable Resource Science, 1-7-22 Suehirocho, Tsurumi-ku, Yokohama,

5 Kanagawa 230-0045, Japan

$6{ }^{2}$ Graduate School of Medical Life Science, Yokohama City University, 1-7-29 Suehirocho,

7 Tsurumi-ku, Yokohama, Kanagawa 230-0045, Japan

$8{ }^{3}$ Graduate School of Bioagricultural Sciences, Nagoya University, 1 Furo-cho, Chikusa-ku,

9 Nagoya, Aichi 464-0810, Japan

$10{ }^{4}$ RIKEN Biomass Engineering Program, 1-7-22 Suehirocho, Tsurumi-ku, Yokohama, Kanagawa

11 230-0045, Japan

12 Taiga Asakura: asakurataiga @tsurumi.yokohama-cu.ac.jp, Kenji Sakata: kenbo@psc.riken.jp,

13 Seiji Yoshida: seiji.y_817@tsurumi.yokohama-cu.ac.jp, Yasuhiro Date: yasuhiro.date@ riken.jp

14 and Jun Kikuchi: jun.kikuchi@riken.jp

$15 *$ Corresponding author

16 Jun Kikuchi: RIKEN Center for Sustainable Resource Science, 1-7-22 Suehirocho, Tsurumi-ku,

17 Yokohama, Kanagawa 230-0045, Japan. Tel: +81455039439; Fax: +81455039489; E-mail:

18 jun.kikuchi@riken.jp 


\section{ABSTRACT}

20 An NMR-based metabolomic approach in aquatic ecosystems is valuable for studying the

21 environmental effects of pharmaceuticals and other chemicals on fish. This technique has also

22 contributed to new information in numerous research areas, such as basic physiology and

23 development, disease, and water pollution. We evaluated the microbial diversity in various fish

24 species collected from Japan's coastal waters using next-generation sequencing, followed by

25 evaluation of the effects of feed type on co-metabolic modulations in fish-microbial symbiotic

26 ecosystems in laboratory-scale experiments. Intestinal bacteria of fish in their natural

27 environment were characterized (using 16S rRNA genes) for trophic level using pyrosequencing

28 and noninvasive sampling procedures developed to study the metabolism of intestinal symbiotic

29 ecosystems in fish reared in their environment. Metabolites in feces were compared, and

30 intestinal contents and feed were annotated based on HSQC and TOCSY using SpinAssign and

31 network analysis. Feces were characterized by species and varied greatly depending on the

32 feeding types. In addition, feces samples demonstrated a response to changes in the time series of

33 feeding. The potential of this approach as a non-invasive inspection technique in aquaculture is

34 suggested. 


\section{PeerJ Reviewing Manuscript}

\section{INTRODUCTION}

36 Most higher organisms such as vertebrates, including humans, harbor a large and diverse number

37 of microorganisms (Sorokin et al. 2008) and enjoy significant benefits from such symbiotic

38 relationships (Backhed et al. 2005). In symbiotic ecosystems, the microbial symbionts facilitate

39 extensive interactive modulation of metabolism and physiology during crosstalk with their hosts.

40 Thereby, they can provide traits that the hosts have not evolved on their own, for example, they

41 may synthesize essential amino acids and vitamins or process otherwise indigestible components

42 in the diet (Gill et al. 2006). Intestinal microbial symbionts often promote nutritional

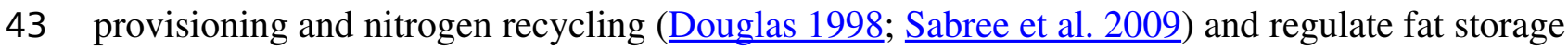

44 for their hosts (Turnbaugh et al. 2006). This demonstrates that microbial symbionts have an

45 important role in maintaining metabolic and physiological homeostasis in these ecosystems.

46 During the investigation of vertebrate intestinal symbionts, mammals such as rodents and humans

47 are commonly studied in terms of microbial structures, diversities, and functions, and metabolic

48 variations and interactions through the crosstalk with hosts; this is despite the fact that mammals

49 comprise fewer than $10 \%$ of total vertebrate diversity. Compared to mammals, fish, originated

50 over 600 million years ago, encompass nearly half the total number of vertebrate species with

51 approximately 28000 extant species (Nelson 2006). Because fish are located near the base of the

52 vertebrate tree of life, investigation of their symbiotic ecosystems are considered important in

53 understanding the co-evolution of mammalian-microbial symbiosis as well as co-metabolic

54 modulations facilitated by the crosstalk between hosts and their microbial symbionts. In this

55 regard, a previous study highlighted a trend of convergent acquisition of similar bacterial

56 communities by fish and mammals, raising the possibility that fish were the first to evolve

57 symbiotic relationships resembling those found among extant gut fermenting mammals ( $\underline{\text { Sullam }}$ 
58 et al. 2012).

59 When studying co-metabolic modulation by hosts and their microbial symbionts, metabolic

60 profiling is a key approach for characterization and evaluation of metabolism and physiology.

61 Using nuclear magnetic resonance (NMR)-based metabolomic (or metabonomic) approaches

62 provides technical insights for characterizing the interactions of hosts and symbionts (Brindle et

63 al. 2002; Li et al. 2008; Nicholson et al. 1999), and this method is capable of generating

64 comparable data between laboratories, thus supporting its continued use (Viant et al. 2009). This

65 approach has many advantages for studying host-microbial interactions and assessing metabolic

66 function and homeostasis at the molecular fingerprinting level (Dumas et al. 2006; Nicholson et

67 al. 1999). Therefore, an NMR-based metabolomic approach has been successfully applied to

68 evaluate animal homeostasis, including in human beings (Claesson et al. 2012; $\underline{\text { Clayton et al. }}$

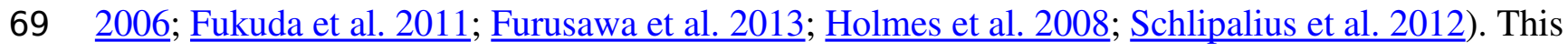

70 NMR-based metabolomic approach is also valuable in aquatic ecosystems for studying the

71 environmental effects of pharmaceuticals and other chemicals on fishes (Samuelsson et al. 2011;

72 Samuelsson et al. 2006; Samuelsson \& Larsson 2008). Such studies have contributed knowledge

73 of basic physiology and development of fish, disease, water pollution, and other aspects

74 (Bilandzic et al. 2011; Dove et al. 2012; $\underline{\text { Picone et al. 2011; }}$ Southam et al. 2008; $\underline{\text { Southam et al. }}$

75 2011; Wagner et al. 2014; Williams et al. 2009). Fish symbiotic ecosystems, however, are rarely

76 investigated, so little information is available concerning metabolic variations associated with

77 fluctuations in microbial composition and structures in diverse fish species affected by the

78 crosstalk between hosts and their microbial symbionts.

79 In the current study, we focus on the characterization and evaluation of metabolic variation and

80 diversity associated with the microbial variation and diversity of symbiotic communities in 
81 diverse fish species. To this end, the metabolic profiling of fish-microbial symbiotic ecosystems

82 was proposed using a methanol-solvent system for an NMR-based metabolomic approach with

83 the network visualization of the spin coupling of NMR spectra in combination with noninvasive

84 and time-course sampling. Furthermore, we evaluated the microbial diversity in various fish

85 species collected from Japan's coastal waters using next-generation sequencing, followed by

86 evaluation of the effects of different feed types on the co-metabolic modulations in fish-microbial

87 symbiotic ecosystems in laboratory-scale experiments (Fig. 1).

\section{MATERIALS AND METHODS}

\section{$89 \quad$ Sample collections and preparations}

90 The fishes used in this study were collected between 2011 and 2013 from estuarine and coastal

91 regions of Kanto, Tohoku, and Southern (Kyusyu-Okinawa) regions. The species of collected

92 fishes, numbers of samples, and their corresponding sampling sites are listed in Table S1 (for

93 sequencing analysis) and S3 (for experiments in rearing environment). The fish samples collected

94 were dissected and gutted. The collected gut contents from the whole intestines were lyophilized

95 and crushed (10 min) to a powder using an Automill machine (Tokken, Inc., Chiba, Japan) and

96 used for microbial community analysis.

97 Metabolic evaluation of intestinal symbiotic ecosystems in artificially controlled

98 environments

99 Fish collected from natural environments were reared in laboratory-scale aquariums under

100 artificially controlled conditions. The aquariums were filled about two thirds in volume with

101 artificially synthesized seawater made from "Instant Ocean" (NAPQO, Ltd, Tokyo, Japan), 
102 equipped with a Protein skimmer (Kamihata, Inc., Hyogo, Japan), air pump (GEX, Inc., Osaka,

103 Japan), water recycling system (EHEIM, Inc., Chiba, Japan), water cooler (ZENSUI, Inc., Osaka,

104 Japan) and maintained at $18-20{ }^{\circ} \mathrm{C}$. The fish used in this experiment and their feeding conditions

105 are described in Table S3. Feces obtained before providing an artificial diet was labeled as "local

106 feces." The fishes were reared under different conditions thereafter. Feed was given to the fish

107 until satiation 3 days a week. Fecal sampling was implemented every day, and samples were

108 collected unbroken in droppers (SANSYO, Inc., Tokyo, Japan). The collected feces samples were

109 lyophilized and crushed (10 min) to a powder using an Automill machine (Tokken, Inc., Chiba,

110 Japan) and used for metabolomic and microbial analysis.

\section{$111 \quad$ NMR measurements}

112 Five milligrams of each of the powdered samples were extracted with $600 \mu \mathrm{L}$ of deuterated

113 methanol solvent with $1 \mathrm{mM}$ of sodium 2,2-dimethyl-2-silapentane-5-sulfonate (DSS) as an

114 internal standard at $60^{\circ} \mathrm{C}$ for $15 \mathrm{~min}$. After centrifugation at $25^{\circ} \mathrm{C}$ for $5 \mathrm{~min}$, the extracted

115 supernatant was transferred to a 5-mm NMR tube for the NMR measurements. One-dimensional

116 (1D) spectra of the samples were acquired at $298 \mathrm{~K}$ using a Bruker AVANCE II 700

117 spectrometer equipped with an ${ }^{1} \mathrm{H}$ inverse triple resonance cryogenically cooled probe with Z-

118 axis gradients (Bruker BioSpin GmbH, Rheinstetten, Germany), as described previously (Date et

119 al. 2014). In brief, ${ }^{1} \mathrm{H}$ NMR spectra were acquired by the standard Bruker pulse program

$120 \mathrm{p} 3919 \mathrm{gp}$, with $32 \mathrm{~K}$ data points with a spectral width of $14 \mathrm{ppm}$, were collected into 32 transients

121 and 8 dummy scans. Prior to Fourier transformation, free induction decays were multiplied by an

122 exponential window function corresponding to a $0.3-\mathrm{Hz}$ line broadening factor. The acquired

123 spectra were manually phased and baseline corrected. 
124 The method for the NMR measurement of the two-dimensional ${ }^{1} \mathrm{H}-{ }^{13} \mathrm{C}$ heteronuclear single

125 quantum coherence (HSQC) and total correlation spectroscopy (TOCSY) has been previously

126 described (Sekiyama et al. 2010; Sekiyama et al. 2011). In the HSQC NMR spectra, a total of

127128 complex $\mathrm{F} 1\left({ }^{13} \mathrm{C}\right)$ and 1024 complex $\mathrm{F} 2\left({ }^{1} \mathrm{H}\right)$ points were recorded from 256 scans per f1

128 increment. The spectral widths were 150 and 14 ppm for F1 and F2, respectively. The TOCSY

129 spectra were acquired in the range of 10.7 to $-1.7 \mathrm{ppm}$ using $4096(\mathrm{~F} 2)$ and $512(\mathrm{~F} 1)$ data points

130 with 16 scans and an interscan delay of $2 \mathrm{~s}$ with 16 dummy scans. The mixing time (D9) was set

131 to $90 \mathrm{~ms}$. The HSQC-TOCSY spectra were acquired as $1024(\mathrm{~F} 2)$ and $256(\mathrm{~F} 1)$ data points with

132180 scans and an interscan delay of $1 \mathrm{~s}$ with 16 dummy scans. The NMR spectra were processed

133 using Bruker TopSpin software (Bruker BioSpin $\mathrm{GmbH}$ ) and assigned using the SpinAssign

134 program at the PRIMe website (http://prime.psc.riken.jp/) (kiyama et al. 2008; $\underline{\text { Chikayama et al. }}$

135 2010; Chikayama et al. 2008) and the Biological Magnetic Resonance Bank

136 (http://www.bmrb.wisc.edu/metabolomics/query metab.php) (Ulrich et al. 2008).

\section{Microbial community analysis}

138 Microbial DNA extraction was performed according to previous studies with slight

139 modifications (Date et al. 2012a). Each DNA sample was amplified by PCR using universal

140 bacterial primers 954f and 1369r targeted to the V6-V8 regions of the 16S rRNA gene according

141 to a previous report (Date et al. 2010). The sequencing analysis and the data processing was

142 outsourced to Operon Biotechnologies Co. Ltd. (Tokyo, Japan). The categorizations of bacterial

143 taxa were performed using a Ribosomal Database Project (RDP;

144 http://rdp.cme.msu.edu/seqmatch/seqmatch intro.jsp) classifier (Wang et al. 2007). 


\section{Statistical analysis}

146 All 1D ${ }^{1} \mathrm{H}-\mathrm{NMR}$ data were reduced by subdividing the spectra into designated sequential 0.01-

147 ppm regions between ${ }^{1} \mathrm{H}$ chemical shifts of 0-9 ppm. After the exclusion of water resonance,

148 each spectrum was normalized by constant sum. Principal component analysis (PCA) was run on

$149 \mathrm{R}$ software and performed according to a previous report (Date et al. 2012b). The data were

150 visualized in the form of the PCA score plots and loading plots. Each data point on the score plots

151 represented an individual sample, and each data point on the loading plots represented one NMR

152 spectral data point related to the metabolites. Partial Least Squares Discriminant Analysis (PLS-

153 DA) was performed on R software with a package "muma." The network diagram was rendered

154 in Gephi (http://gephi.org) according to previous studies (Ito et al. 2014; Yamazawa et al. 2013).

\section{RESULTS AND DISCUSSION}

156 Microbial diversity in gut symbiotic ecosystems of various fish species

157 This study focused on microbiota and metabolic variation of gut contents in various fish species

158 living in estuarine and coastal regions of Japan. To evaluate the diversity of gut microbiota, the

159 microbial composition and structure of the gut contents of various fish species listed in Table S1

160 were analyzed using a next-generation sequencer. Almost all fishes had common bacterial Phyla

161 such as Phylum Proteobacteria, Phylum Firmicutes, and Phylum Actinobacteria as dominant

162 bacterial categories with some exceptions (Fig. S1). In order to compare the microbiota profiles

163 among fishes, PCA was performed on the sequencing data (Fig. S2 and S3). From the PCAs,

164 omnivorous fish were likely to be located on the negative PC1 side, whereas carnivorous fish

165 were likely to be located on the positive PC1 side. The analysis of diversity index on the basis of

166 the Shannon index showed that the trend for omnivorous fish was toward high diversity and was 
167 located on the negative PC2 side, whereas the profiles with relatively low diversity index values

168 were located on the positive PC2 side (Fig. S2A). Diadromous fish tended to be clustered on the

169 negative PC4 side (Fig. S2B). Based on the loading plot analyses, the factors contributing to

170 omnivorous feeding habitat clusters were shown as, e.g., unclassified Hyphomicrobiaceae

171 bacterium, Mycobacterium sp., and Ilumatobacter sp., whereas the factors contributing to

172 carnivorous feeding habitat clusters were shown as, e.g., Methylobacterium sp., Bacillus sp., and

173 Arthrobacter sp. (Fig. S2C). The contributing factors for the fishes showing low diversity index

174 values in their guts were shown as, e.g., unclassified Vibrionaceae bacterium and Clostridium sp.,

175 whereas the contributing factors for diadromous fish were shown as, e.g., Deinococcus sp. and

176 unclassified Micrococcaceae bacterium (Fig. S2D and S2E). Fish with differing feeding habits

177 have characteristic microbiota; therefore, it is possible to characterize the fish according to their

178 intestinal microbiota. These changes were expected to affect the physiology and health of the

179 fishes, and therefore we performed the metabolomic evaluation using a diverse range of fishes

180 selected from the above species.

\section{Metabolic characterization of gut content and feces using methanol solvent system}

182 In aquafarming industries, non-invasive sampling to evaluate the health and physiological effects

183 of the environment on fish should be possible and would contribute significantly to the farming

184 process. Thus, we focused on metabolic profiling of fish feces for evaluating health and

185 physiological variation. For this purpose, the water extraction method for NMR measurements is

186 important when similar metabolomics are performed on humans and mice, however, because

187 sampling in water is unavoidable with fish, this caused concern that many water-soluble

188 components may be diffusing into the water. Therefore, in the present study, we analyzed the 
189 fractions obtained using methanol solvent, and the metabolic variability of the fractions was

190 evaluated by ${ }^{1} \mathrm{H}$ NMR spectra of feces of 14 fish (Fig. S1). For the annotation of metabolites

191 extracted from methanol solvents, we measured mixtures of three diets (Polychaeta, Crustacea,

192 and Aquaculture feeds), feces, and intestinal contents of Epinephelus septemfasciatus and used

193 our originally customized database (SpinAssign program) for HSQC annotations with the

194 network visualization of the spin coupling on TOCSY and HSQC-TOCSY NMR spectra to

195 enhance the annotation of metabolites (Figs. 3, S5, and S6, and Table S2). However, the

196 methanol solvent also extracts polar metabolites that are water-soluble, and therefore, the

197 retention capability of water-soluble components in feces was evaluated by a water rinse

198 experiment for fish feces. The collected feces were rinsed three times with artificial seawater for

19915 min with shaking. The fecal samples before and after rinsing were extracted with methanol

200 and measured by NMR spectroscopy. Almost all signals excluding leucine detected in HSQC

201 NMR spectra were retained after rinsing, as shown in Figure S6. In particular, water-soluble

202 components such as amino acids and sugars were detected in feces by HSQC spectra using

203 methanol extraction (Figs. S5 and S6). The retention of water-soluble components may be

204 because of a physical property of feces, i.e., the water-soluble components may be packed and 205 aggregated in feces either chemically (by chemical and physical interactions) and/or biologically

206 (by biological interactions such as retention in cells and/or trapping by microbes).

207 Comparisons of microbial and metabolic profiles among diets, intestinal contents, and 208 feces of fish

209 To evaluate the microbiota and metabolic profiles of gut contents and feces in a rearing 210 environment, sequencing analysis and ${ }^{1} \mathrm{H}$ NMR-based metabolic profiling were performed in 
211 combination with evaluation by PCA (Fig. S7). The microbiota and metabolic profiles in feces

212 showed trends of clustering on the basis of feeding conditions, whereas those profiles in gut

213 contents showed no clear clustering under the feeding conditions. This result indicated that the

214 feces as an aggregate of final metabolic products were influenced by feedings compared with gut

215 contents. It also suggested covariations between the microbial community and metabolites in

216 feces and gut contents on the basis of the differences in feeding conditions. In addition, on the

217 basis of the intensities of the annotated peaks evaluated by HSQC and TOCSY spectra using the

218 network visualization approach, we further analyzed and compared the metabolic profiles among

219 diets, intestinal contents, and feces of fish (Fig. 4). The concentrations of these samples were

220 uniform in $50 \mathrm{mg}$ of feces/600 $\mu \mathrm{l}$ of MeOD and were normalized by the internal standard ( $1 \mathrm{mM}$

221 DSS). They were compared in relative amounts on the basis of the peak of feeds (as 1.0) in Fig.

222 3. Sugar, lipid, and peptide peaks were commonly observed in data from the feeds. A relatively

223 high number of lipid peaks were observed in feces samples and intestinal contents, whereas the

224 peaks from sugars and peptides were reduced. Many peaks detected in the intestinal contents

225 were no longer present in the feces samples. Based on the peak intensity of the individual

226 metabolites, reduction rates were evaluated for the intestinal contents and the fish feces for each

227 feed type. The levels of evaluated metabolites decreased in the change from intestinal contents to

228 feces for each feed type and were lowest in the feces. The reduction rate was characterized by the

229 respective substances. Assuming that feeds are 100\%, the phospholipids and sugars were reduced

230 to less than $30 \%$ by the intestinal content stage, and had become approximately $10 \%$ in the feces.

231 In contrast, the lipid peak was more than $40 \%$ in the intestinal contents and $20 \%$ in the feces. It

232 was shown that absorption rates of each metabolite had a characteristic profile. Further, the

233 metabolites that were difficult to decompose remained in the intestines and were lost in the feces; 
234 they were considered to be incompletely absorbed by host fish species and unavailable by

235 intestinal microbiota. From these results we can see that metabolic information about the fishes

236 was reflected in the methanol fraction of the feces.

237 Comparisons of metabolic diversity of various fish species under artificially controlled

238 environments

239 To evaluate the metabolic dynamics in intestinal content in various fish species and to

240 characterize the effects of a change in diet on fish-microbial symbiotic ecosystems over time

241 course variations, we focused on the effects of different feed types on the co-metabolic

242 modulations in fish-microbial symbiotic ecosystems in laboratory-scale experiments. For this

243 experiment, 18 species of fish were selected including Acanthogobius flavimanus, Sebastes

244 ventricosus, Sebastiscus marmoratus, and Sillago japonica; the rearing conditions for each fish

245 are listed in Table S3. The different metabolic profiles depending on the fish species were

246 obtained by ${ }^{1} \mathrm{H}$ NMR measurements (Fig. S4). In the evaluation of PCA, the metabolic profiles of

247 the 18 fish species appeared to be largely changed by feeding situation rather than by fish species

248 (Fig. 6A). By loading plot analyses, some metabolites such as fatty acids and phospholipids

249 contributed to the clustering of aquaculture feeds (Fig. 6B). In feces from the rearing

250 environment of Acanthogobius flavimanus, for example, phospholipid, fatty acid, and

251 trimethylamine were increased. Local (natural environment) feces were diffused near the center;

252 it can be seen that the diversity was greater than that in the breeding environment. In addition,

253 there were trends, such as phospholipids and amino acids in local feces were lower than those of

254 the breeding environment from the loading plot analysis. This trend was speculated to result from

255 differences in the composition and digestibilities of protein and lipids in live food and in artificial 
256 diets. Profiling of fish reared on the same feed was strongly clustered, and there was a tendency

257 to cluster by species. In addition, the digestion and absorption of feed in different species was

258 reflected in their feces.

259 Time-series behavior based on differences of feeding in the same species, were evaluated by

260 profiling methods described above in the rearing environment. Results of PCA show that feces

261 profile varied greatly but temporarily due to a change in feed and tended to return to the original

262 profile after a period of time (Fig. 5A). Based on the loading plot analysis, some metabolites such

263 as phospholipids and fatty acids contributed to the variations by the impact of aquaculture

264 feeding (Fig. 5B). In addition, microbiota profiles widely varied with the effect of feeding change

265 by aquaculture feed, but the community was likely to be restored when carnivorous feeding was

266 again available to the fishes (Fig. 5C). This result suggests that feeding changes in Epinephelus

267 septemfasciatus affected the variations in metabolic and microbiota profiles in feces. In these

268 results, a difference in response to time series of feeding associated with fecal metabolic and

269 microbiota profiles can be observed. With information of the intestinal environment being

270 reflected in the fish feces, we propose that this could be an informative non-invasive technique

271 for cultured fish.

272 In this study, we evaluated the metabolic and microbiota profiles in fish-microbial symbiotic

273 ecosystems. To evaluate variations in metabolism of intestinal symbiotic ecosystems using

274 noninvasive sampling, one of the disadvantages was the water-solubility of components of the

275 fish feces due to their aquatic environment. To circumvent this issue, we applied the methanol-

276 solvent-based metabolomic approach with a propose that metabolic and microbiota profiling in

277 fish feces using the methanol solvent system could be an informative noninvasive technique for 
278 cultured fish. The application of our approach is a step toward clarifying the metabolic dynamics

279 of the complex intestinal microbial community living inside fish in aquatic environments. It also

280 translates microbial activity and function into host responses, and may even provide a means to

281 engineer the metabolic activities of intestinal microorganisms to improve the health of fish in the

282 form of prebiotic or probiotic treatments in the aquafarming industry.

283 In summary, this study demonstrates the metabolomic analysis of fish feces with noninvasive

284 sampling using methanol-solvent systems with the network visualization approach based on

285 TOCSY spectra in combination with HSQC peak lists annotated by the SpinAssign program.

286 Using this approach, we evaluated the metabolic diversity of various fish species from coastal

287 environments around Japan while they were in laboratory-scale rearing systems and revealed that

288 the diversity was caused by differences in the feeding situations rather than the diversity of fish

289 species.

\section{ACKNOWLEDGMENTS}

291 The authors wish to thank Yuuri Tsuboi, Amiu Shino (RIKEN), and Yuho Sato (Yokohama

292 City University) for stimulating discussions, technical assistance, and useful advice on NMR 293 measurements and analysis.

\section{References}

295

296

297

298

299

300

301

302
Akiyama K, Chikayama E, Yuasa H, Shimada Y, Tohge T, Shinozaki K, Hirai MY, Sakurai T, Kikuchi J, and Saito K. 2008. PRIMe: a Web site that assembles tools for metabolomics and transcriptomics. In Silico Biol 8:339-345.

Backhed F, Ley RE, Sonnenburg JL, Peterson DA, and Gordon JI. 2005. Host-bacterial mutualism in the human intestine. Science 307:1915-1920.

Bilandzic N, Dokic M, and Sedak M. 2011. Metal content determination in four fish species from the Adriatic Sea. Food Chemistry 124:1005-1010.

Brindle JT, Antti H, Holmes E, Tranter G, Nicholson JK, Bethell HW, Clarke S, Schofield PM, 
McKilligin E, Mosedale DE, and Grainger DJ. 2002. Rapid and noninvasive diagnosis of the presence and severity of coronary heart disease using $1 \mathrm{H}-\mathrm{NMR}$-based metabonomics. Nat Med 8:1439-1444.

Chikayama E, Sekiyama Y, Okamoto M, Nakanishi Y, Tsuboi Y, Akiyama K, Saito K, Shinozaki $\mathrm{K}$, and Kikuchi J. 2010. Statistical indices for simultaneous large-scale metabolite detections for a single NMR spectrum. Anal Chem 82:1653-1658.

Chikayama E, Suto M, Nishihara T, Shinozaki K, and Kikuchi J. 2008. Systematic NMR analysis of stable isotope labeled metabolite mixtures in plant and animal systems: coarse grained views of metabolic pathways. PLoS One 3:e3805.

Claesson MJ, Jeffery IB, Conde S, Power SE, O'Connor EM, Cusack S, Harris HM, Coakley M, Lakshminarayanan B, O'Sullivan O, Fitzgerald GF, Deane J, O'Connor M, Harnedy N, O'Connor K, O'Mahony D, van Sinderen D, Wallace M, Brennan L, Stanton C, Marchesi JR, Fitzgerald AP, Shanahan F, Hill C, Ross RP, and O'Toole PW. 2012. Gut microbiota composition correlates with diet and health in the elderly. Nature 488:178-184.

Clayton TA, Lindon JC, Cloarec O, Antti H, Charuel C, Hanton G, Provost JP, Le Net JL, Baker D, Walley RJ, Everett JR, and Nicholson JK. 2006. Pharmaco-metabonomic phenotyping and personalized drug treatment. Nature 440:1073-1077.

Date Y, likura T, Yamazawa A, Moriya S, and Kikuchi J. 2012a. Metabolic sequences of anaerobic fermentation on glucose-based feeding substrates based on correlation analyses of microbial and metabolite profiling. Journal of Proteome Research 11:56025610.

Date Y, Nakanishi Y, Fukuda S, Kato T, Tsuneda S, Ohno H, and Kikuchi J. 2010. New monitoring approach for metabolic dynamics in microbial ecosystems using stableisotope-labeling technologies. Journal of Bioscience and Bioengineering 110:87-93.

Date Y, Nakanishi Y, Fukuda S, Nuijima Y, Kato T, Umehara M, Ohno H, and Kikuchi J. 2014. In vitro evaluation method for screening of candidate prebiotic foods. Food Chemistry 152:251-260.

Date Y, Sakata K, and Kikuchi J. 2012b. Chemical profiling of complex biochemical mixtures from various seaweeds. Polymer Journal 44:888-894.

Douglas AE. 1998. Nutritional interactions in insect-microbial symbioses: aphids and their symbiotic bacteria Buchnera. Annu Rev Entomol 43:17-37.

Dove AD, Leisen J, Zhou M, Byrne JJ, Lim-Hing K, Webb HD, Gelbaum L, Viant MR, Kubanek J, and Fernandez FM. 2012. Biomarkers of whale shark health: a metabolomic approach. PLoS One 7:e49379.

Dumas ME, Maibaum EC, Teague C, Ueshima H, Zhou B, Lindon JC, Nicholson JK, Stamler J, Elliott P, Chan Q, and Holmes E. 2006. Assessment of analytical reproducibility of $1 \mathrm{H}$ NMR spectroscopy based metabonomics for large-scale epidemiological research: the INTERMAP Study. Anal Chem 78:2199-2208.

Fukuda S, Toh H, Hase K, Oshima K, Nakanishi Y, Yoshimura K, Tobe T, Clarke JM, Topping DL, Suzuki T, Taylor TD, Itoh K, Kikuchi J, Morita H, Hattori M, and Ohno H. 2011. Bifidobacteria can protect from enteropathogenic infection through production of acetate. Nature 469:543-547.

Furusawa Y, Obata Y, Fukuda S, Endo TA, Nakato G, Takahashi D, Nakanishi Y, Uetake C, Kato K, Kato T, Takahashi M, Fukuda NN, Murakami S, Miyauchi E, Hino S, Atarashi K, Onawa S, Fujimura Y, Lockett T, Clarke JM, Topping DL, Tomita M, Hori S, Ohara O, Morita T, Koseki H, Kikuchi J, Honda K, Hase K, and Ohno H. 2013. Commensal microbe-derived butyrate induces the differentiation of colonic regulatory $\mathrm{T}$ cells. Nature 504:446-450.

Gill SR, Pop M, Deboy RT, Eckburg PB, Turnbaugh PJ, Samuel BS, Gordon Jl, Relman DA, Fraser-Liggett CM, and Nelson KE. 2006. Metagenomic analysis of the human distal gut microbiome. Science 312:1355-1359.

Holmes E, Loo RL, Stamler J, Bictash M, Yap IK, Chan Q, Ebbels T, De lorio M, Brown IJ, Veselkov KA, Daviglus ML, Kesteloot H, Ueshima H, Zhao L, Nicholson JK, and Elliott P. 2008. Human metabolic phenotype diversity and its association with diet and blood pressure. Nature 453:396-400.

Ito K, Sakata K, Date Y, and Kikuchi J. 2014. Integrated analysis of seaweed components during seasonal fluctuation by data mining across heterogeneous chemical measurements with network visualization. Analytical Chemistry 86:1098-1105. 
361

362

363

364

365

366

367

368

369

370

371

372

373

374

375

376

377

378

379

380

381

382

383

384

385

386

387

388

389

390

391

392

393

394

395

396

397

398

399

400

401

402

403

404

405

406

407

408

409

410

411

412

413

414

415

416

417

418

Li M, Wang B, Zhang M, Rantalainen $M$, Wang S, Zhou H, Zhang $Y$, Shen J, Pang X, Zhang $M$, Wei H, Chen Y, Lu H, Zuo J, Su M, Qiu Y, Jia W, Xiao C, Smith LM, Yang S, Holmes E, Tang H, Zhao G, Nicholson JK, Li L, and Zhao L. 2008. Symbiotic gut microbes modulate human metabolic phenotypes. Proc Natl Acad Sci U S A 105:2117-2122. Nelson JS. 2006. Fishes of the World, 4th Edition.

Nicholson JK, Lindon JC, and Holmes E. 1999. 'Metabonomics': understanding the metabolic responses of living systems to pathophysiological stimuli via multivariate statistical analysis of biological NMR spectroscopic data. Xenobiotica 29:1181-1189.

Picone G, Engelsen SB, Savorani F, Testi S, Badiani A, and Capozzi F. 2011. Metabolomics as a Powerful Tool for Molecular Quality Assessment of the Fish Sparus aurata. Nutrients 3:212-227.

Sabree ZL, Kambhampati S, and Moran NA. 2009. Nitrogen recycling and nutritional provisioning by Blattabacterium, the cockroach endosymbiont. Proc Natl Acad Sci U S A 106:19521-19526.

Samuelsson LM, Bjorlenius B, Forlin L, and Larsson DG. 2011. Reproducible (1)H NMR-based metabolomic responses in fish exposed to different sewage effluents in two separate studies. Environ Sci Technol 45:1703-1710.

Samuelsson LM, Forlin L, Karlsson G, Adolfsson-Eric M, and Larsson DGJ. 2006. Using NMR metabolomics to identify responses of an environmental estrogen in blood plasma of fish. Aquatic Toxicology 78:341-349.

Samuelsson LM, and Larsson DG. 2008. Contributions from metabolomics to fish research. Mol Biosyst 4:974-979.

Schlipalius DI, Valmas N, Tuck AG, Jagadeesan R, Ma L, Kaur R, Goldinger A, Anderson C, Kuang J, Zuryn S, Mau YS, Cheng Q, Collins PJ, Nayak MK, Schirra HJ, Hilliard MA, and Ebert PR. 2012. A core metabolic enzyme mediates resistance to phosphine gas. Science 338:807-810.

Sekiyama Y, Chikayama E, and Kikuchi J. 2010. Profiling polar and semipolar plant metabolites throughout extraction processes using a combined solution-state and high-resolution magic angle spinning NMR approach. Analytical Chemistry 82:16431652.

Sekiyama Y, Chikayama E, and Kikuchi J. 2011. Evaluation of a semipolar solvent system as a step toward heteronuclear multidimensional NMR-based metabolomics for 13Clabeled bacteria, plants, and animals. Analytical Chemistry 83:719-726.

Sorokin DY, Tourova TP, Muyzer G, and Kuenen GJ. 2008. Thiohalospira halophila gen. nov., sp. nov. and Thiohalospira alkaliphila sp. nov., novel obligately chemolithoautotrophic, halophilic, sulfur-oxidizing gammaproteobacteria from hypersaline habitats. Int J Syst Evol Microbiol 58:1685-1692.

Southam AD, Easton JM, Stentiford GD, Ludwig C, Arvanitis TN, and Viant MR. 2008. Metabolic Changes in Flatfish Hepatic Tumours Revealed by NMR-Based Metabolomics and Metabolic Correlation Networks. Journal of Proteome Research 7:5277-5285.

Southam AD, Lange A, Hines A, Hill EM, Katsu Y, Iguchi T, Tyler CR, and Viant MR. 2011. Metabolomics Reveals Target and Off-Target Toxicities of a Model Organophosphate Pesticide to Roach (Rutilus rutilus): Implications for Biomonitoring. Environ Sci Technol 45:3759-3767.

Sullam KE, Essinger SD, Lozupone CA, O'Connor MP, Rosen GL, Knight R, Kilham SS, and Russell JA. 2012. Environmental and ecological factors that shape the gut bacterial communities of fish: a meta-analysis. Mol Ecol 21:3363-3378.

Turnbaugh PJ, Ley RE, Mahowald MA, Magrini V, Mardis ER, and Gordon JI. 2006. An obesityassociated gut microbiome with increased capacity for energy harvest. Nature 444:1027-1031.

Ulrich EL, Akutsu H, Doreleijers JF, Harano Y, loannidis YE, Lin J, Livny M, Mading S, Maziuk D, Miller Z, Nakatani E, Schulte CF, Tolmie DE, Kent Wenger R, Yao H, and Markley JL. 2008. BioMagResBank. Nucleic Acids Res 36:D402-408.

Viant MR, Bearden DW, Bundy JG, Burton IW, Collette TW, Ekman DR, Ezernieks V, Karakach TK, Lin CY, Rochfort S, de Ropp JS, Teng Q, Tjeerdema RS, Walter JA, and Wu H. 2009. International NMR-based environmental metabolomics intercomparison exercise. Environ Sci Technol 43:219-225.

Wagner L, Trattner S, Pickova J, Gomez-Requeni P, and Moazzami AA. 2014. H-1 NMR-based 
metabolomics studies on the effect of sesamin in Atlantic salmon (Salmo salar). Food

421

422

423

424

425

426

427

428

429

430

431 Chemistry 147:98-105.

Wang Q, Garrity GM, Tiedje JM, and Cole JR. 2007. Naive Bayesian classifier for rapid assignment of rRNA sequences into the new bacterial taxonomy. Appl Environ Microbiol 73:5261-5267.

Williams TD, Wu HF, Santos EM, Ball J, Katsiadaki I, Brown MM, Baker P, Ortega F, Falciani F, Craft JA, Tyler CR, Chipman JK, and Viant MR. 2009. Hepatic Transcriptomic and Metabolomic Responses in the Stickleback (Gasterosteus aculeatus) Exposed to Environmentally Relevant Concentrations of Dibenzanthracene. Environ Sci Technol 43:6341-6348.

Yamazawa A, Date Y, Ito K, and Kikuchi J. 2014. Visualizing microbial dechlorination processes in underground ecosystem by statistical correlation and network analysis approach. Journal of Bioscience and Bioengineering. 3:305-309. 
433 Figure 1. Conceptual diagram illustrating evaluation strategy of metabolic and microbial 434 dynamics in intestinal environment of fishes using 16S rRNA gene sequence analysis and nuclear 435 magnetic resonance (NMR) with multivariate analysis.

436 Figure 2. Advanced analytical approach using a network visualization method based on TOCSY 437 spectra. (Upper left) Representative TOCSY NMR spectra for methanol-soluble components in 438 fish feces. (Upper right) Visualization of spin coupling network of TOCSY spectra based on 439 correlation network analysis. Green indicates a low magnetic field and red indicates a high 440 magnetic field in ${ }^{1} \mathrm{H}$ NMR chemical shift. The nodes are shown as individual ${ }^{1} \mathrm{H}$ NMR spectral 441 peaks, and lines are drawn on the basis of the cross-peaks of TOCSY spectra.

442 Figure 3. ${ }^{1} \mathrm{H}_{-}{ }^{13} \mathrm{C}$ HSQC spectra of methanol-soluble component from fish feeds, intestinal 443 contents, and feces. Spectral intensities were normalized to an internal standard (DSS). Red, 444 amino acids; blue, unsaturated fatty acids; aqua, fatty acids and phospholipids; green, 445 phospholipids; pink, sugars.

446 Figure 4. Comparisons of spectral intensities in each detected metabolite among Epinephelus 447 septemfasciatus feces, intestinal contents, and feeds. Relative peak intensities of the feces and 448 intestinal contents from fish on the basis of the intensities of feeds are shown. Peak numbers and 449 annotated metabolites are listed in Supplementary Fig. S5 and Table S3. (A) amino acid, (B) 450 unsaturated fatty acid, fatty acid, and phospholipid, (C) sugar region peaks. 
451 Figure 5. Metabolic variations based on ${ }^{1} \mathrm{H}$ NMR profiling of various fishes evaluated by PCA.

452 (A) PCA score plot for ${ }^{1} H$ NMR profiles $\left(k=793, R^{2} X=0.268, R^{2} Y=0.181, Q^{2}=0.201\right)$ of fish 453 feces was computed from 169 samples from 21 species. Letters (a-u) represent fishes listed in 454 Table S3. (B) PCA loading plots on ${ }^{1} \mathrm{H}$ NMR profiles.

455 Figure 6. Metabolic and microbial profiles of feeding response in Epinephelus septemfasciatus 456 feces. Symbols representing individual communities are colored by diet (red: carnivorous 457 feeding, green: aquaculture feeding). Three Epinephelus septemfasciatus were bred by 458 carnivorous feeding for 3 months. They were then divided into three tanks $\left({ }^{*},{ }_{,}^{\dagger}\right)$, and bred for 3 459 weeks by aquaculture feeding. One E. septemfasciatus was then bred with aquaculture feeding, 460 and the others were bred with carnivorous feeding. (A) PCA score plot on ${ }^{1} \mathrm{H}$ NMR profile from 461 feces $\left(n=38, k=808, R^{2} X=0.488, R^{2} Y=0.187, Q^{2}=0.442\right)$. (B) PCA loading plots on ${ }^{1} H$ 462 NMR profiles. Letters S, U, P, A, F, L, I, and V indicate sugar, unsaturated fatty acid, 463 phospholipids, amino acids, fatty acids, leucine, isoleucine, and valine, respectively. (C) Bacterial 464 profile that changed characteristically during the 4 months of rearing. These data were first 465 normalized by total reads for each sample (i.e., the ratio in each fecal sample), and then 466 secondarily normalized on the basis of the sum of the ratios of the same bacteria. 


\section{Figure 1}

Figure 1

Conceptual diagram illustrating evaluation strategy of metabolic and microbial dynamics in intestinal environment of fishes using 16S rRNA gene sequence analysis and nuclear magnetic resonance (NMR) with multivariate analysis.

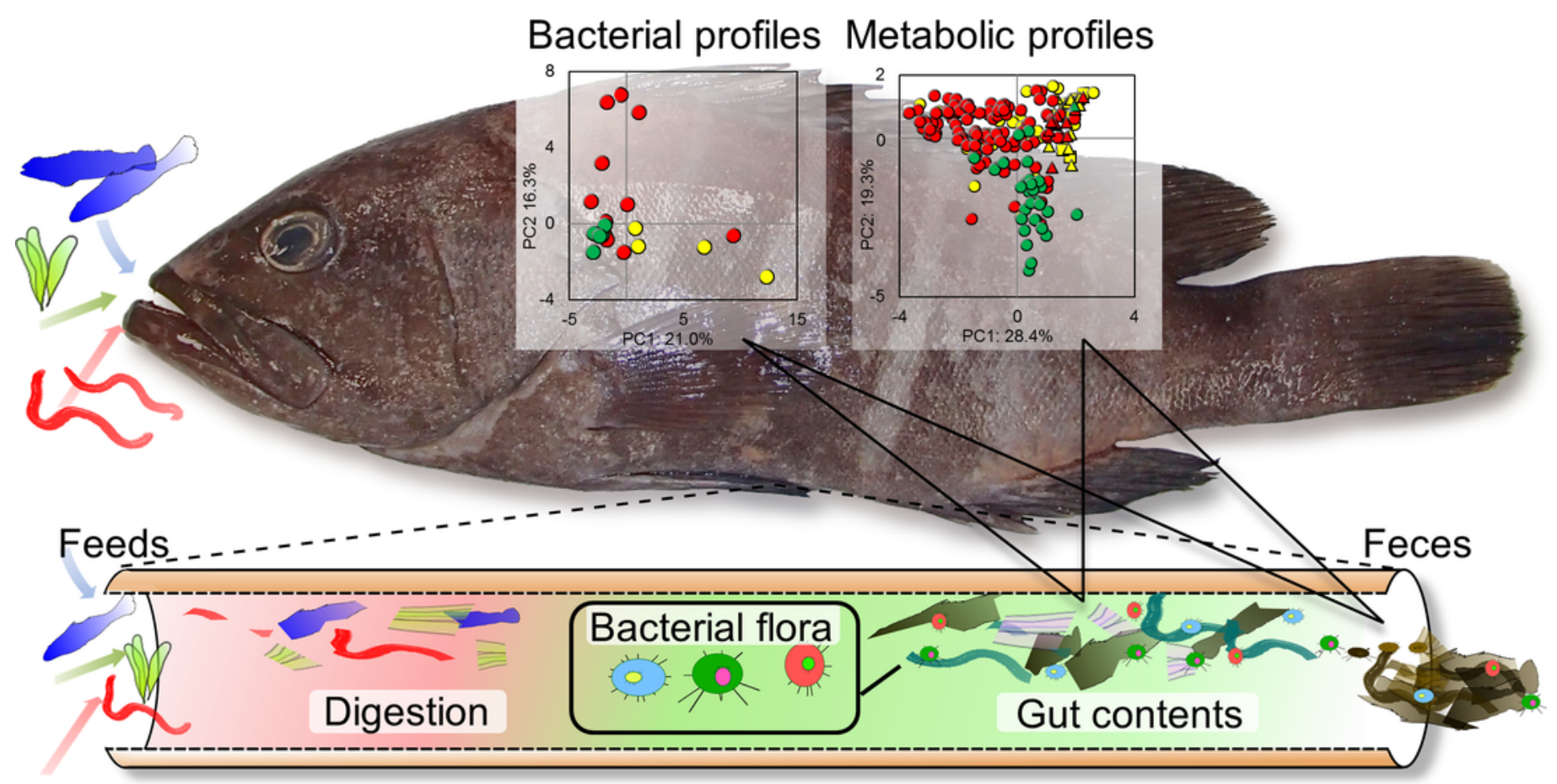




\section{Figure 2}

Figure 2.

Advanced analytical approach using a network visualization method based on TOCSY spectra. (Upper left) Representative TOCSY NMR spectra for methanol-soluble components in fish feces. (Upper right) Visualization of spin coupling network of TOCSY spectra based on correlation network analysis. Green indicates a low magnetic field and red indicates a high magnetic field in ${ }^{1} \mathrm{H}$ NMR chemical shift. The nodes are shown as individual ${ }^{1} \mathrm{H}$ NMR spectral peaks, and lines are drawn on the basis of the cross-peaks of TOCSY spectra. 


\section{Network visualization}

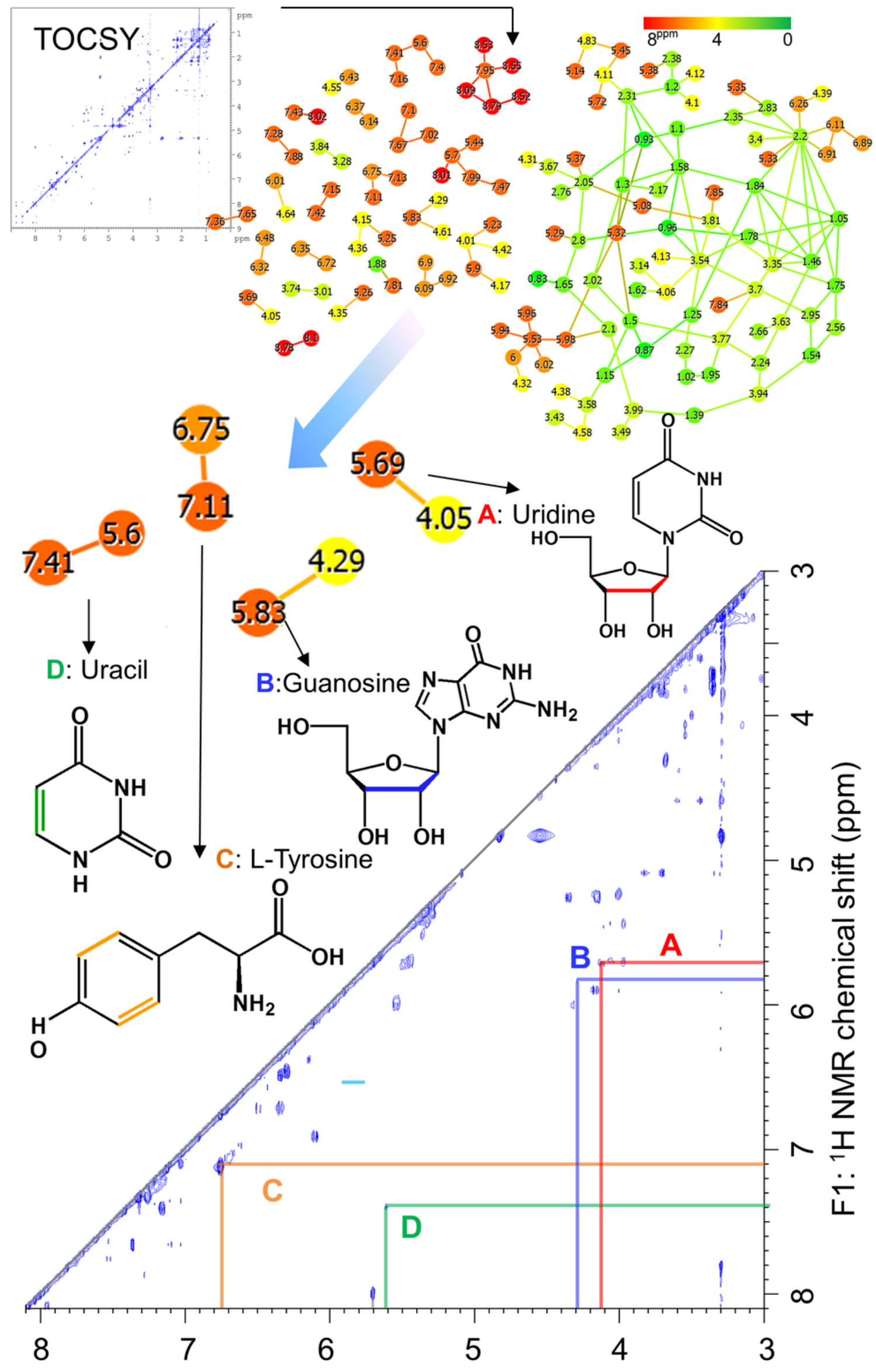




\section{Figure 3}

Figure 3.

${ }^{1} \mathrm{H}-{ }^{13} \mathrm{C}$ HSQC spectra of methanol-soluble component from fish feeds, intestinal contents, and feces. Spectral intensities were normalized to an internal standard (DSS). Red, amino acids; blue, unsaturated fatty acids; aqua, fatty acids and phospholipids; green, phospholipids; pink, sugars. 
Feeds

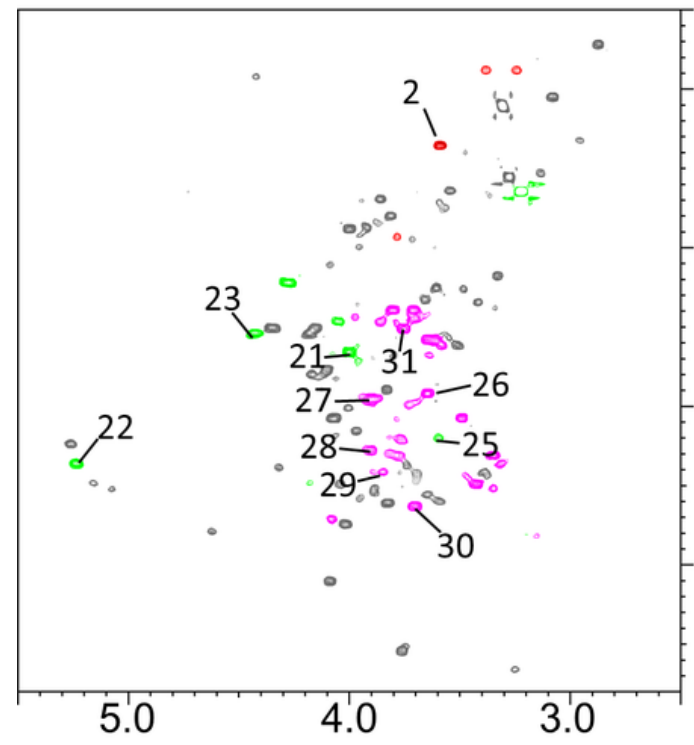

Gut contents

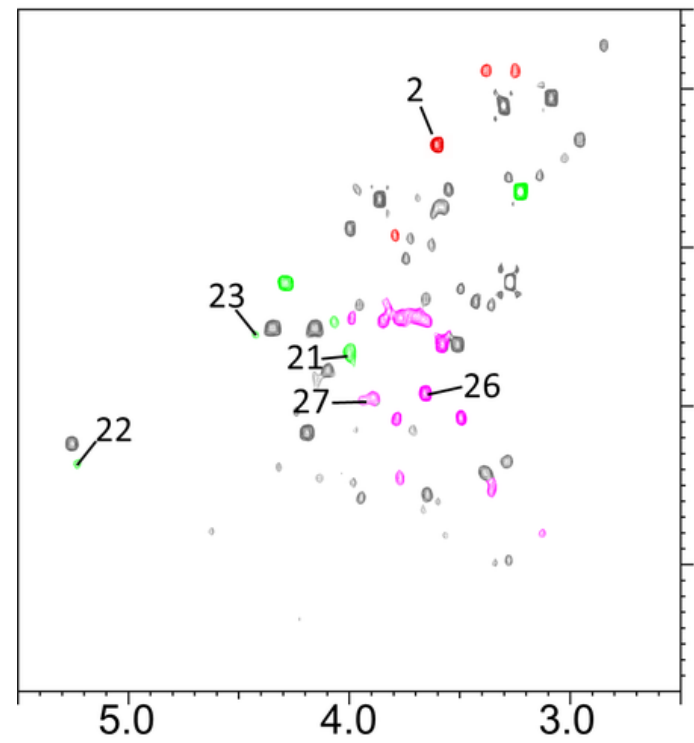

Feces

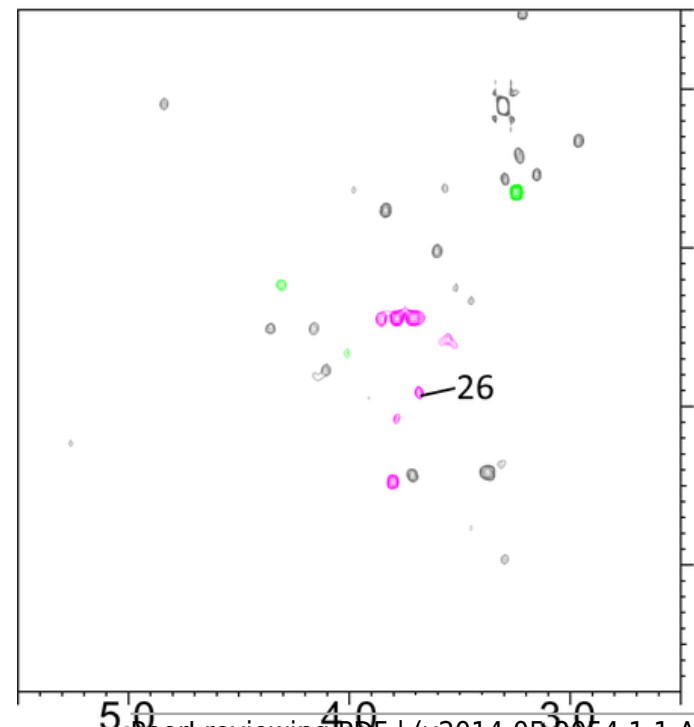

Feeds

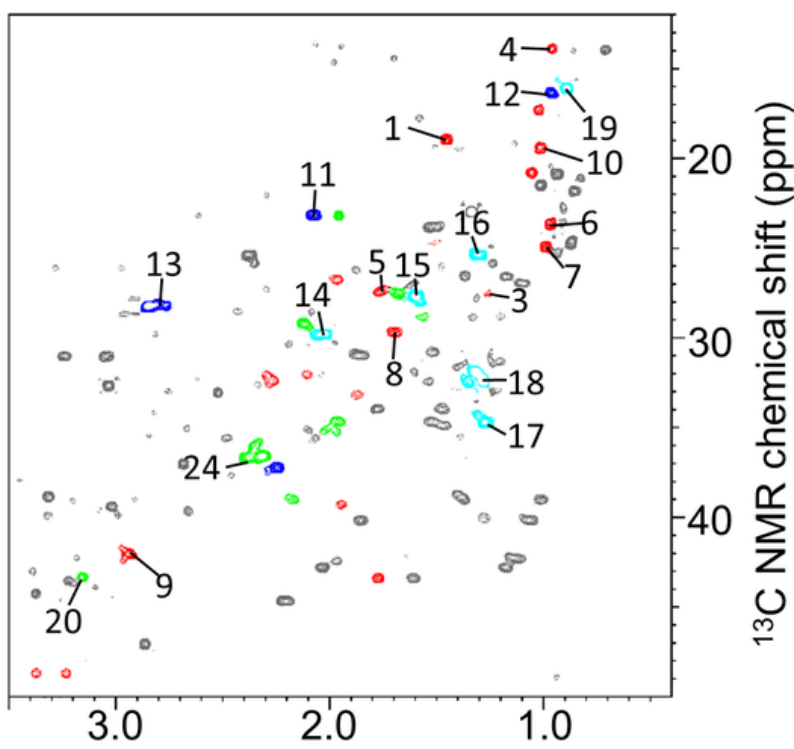

Gut contents

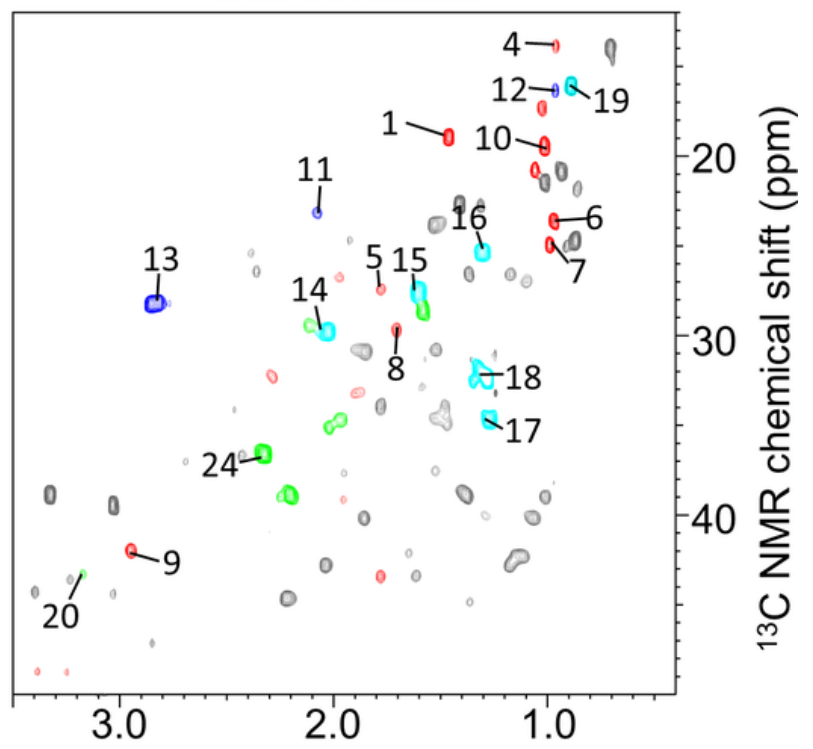

Feces

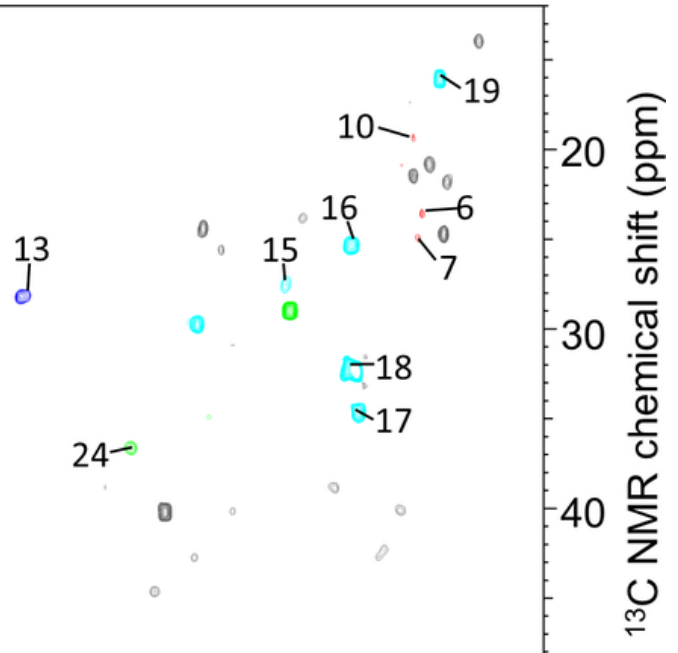




\section{Figure 4}

Figure 4.

Comparisons of spectral intensities in each detected metabolite among Epinephelus septemfasciatus feces, intestinal contents, and feeds. Relative peak intensities of the feces and intestinal contents from fish on the basis of the intensities of feeds are shown. Peak numbers and annotated metabolites are listed in Supplementary Fig. S5 and Table S3. (A) amino acid, (B) unsaturated fatty acid, fatty acid, and phospholipid, (C) sugar region peaks. 


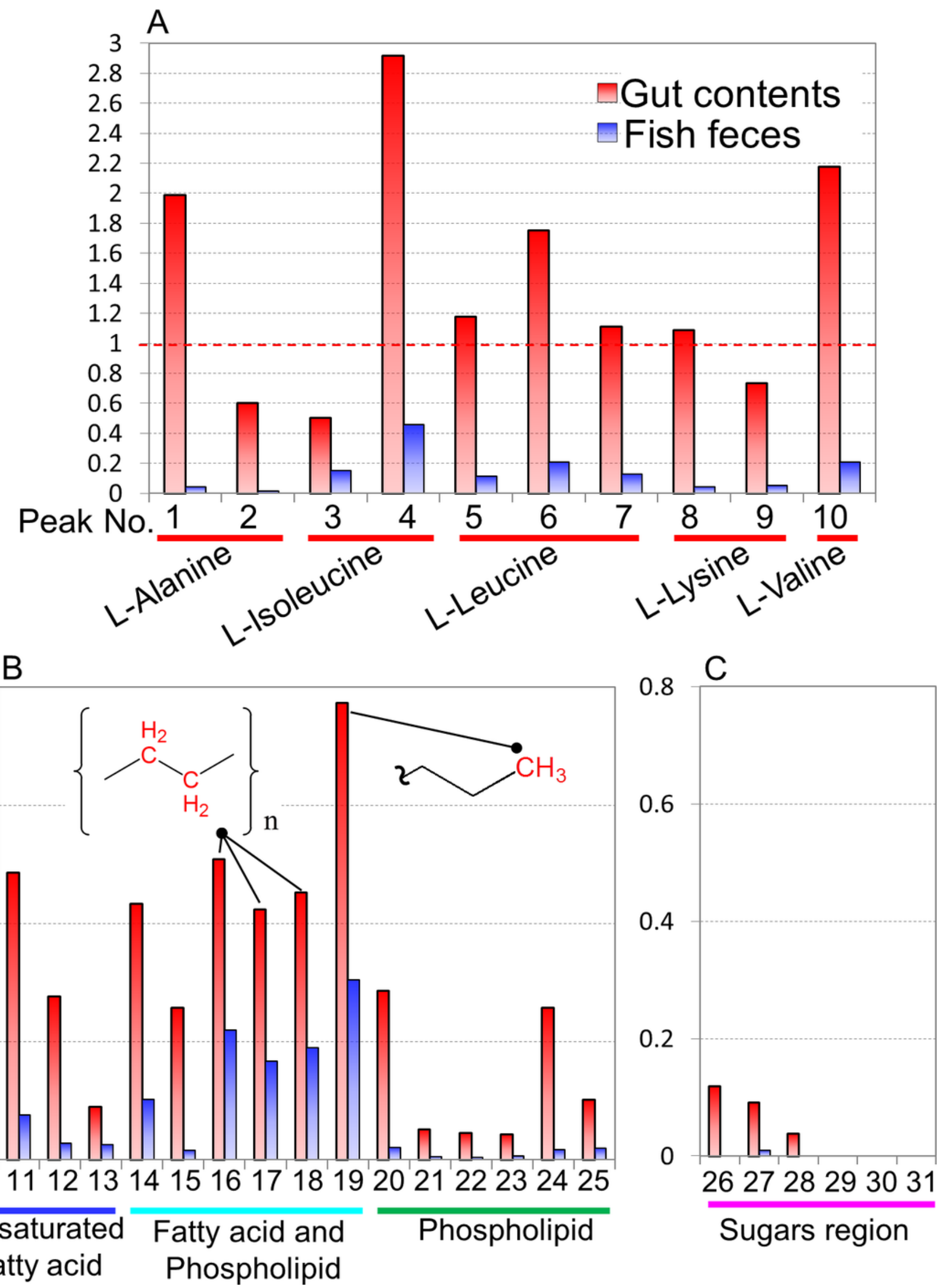


Figure 5

Figure 5.

Metabolic variations based on ${ }^{1} \mathrm{H}$ NMR profiling of various fishes evaluated by PCA. (A) PCA score plot for ${ }^{1} H$ NMR profiles $\left(k=793, R^{2} X=0.268, R^{2} Y=0.181, Q^{2}=0.201\right)$ of fish feces was computed from 169 samples from 21 species. Letters $(a-u)$ represent fishes listed in Table S3. (B) PCA loading plots on ${ }^{1} \mathrm{H}$ NMR profiles. 


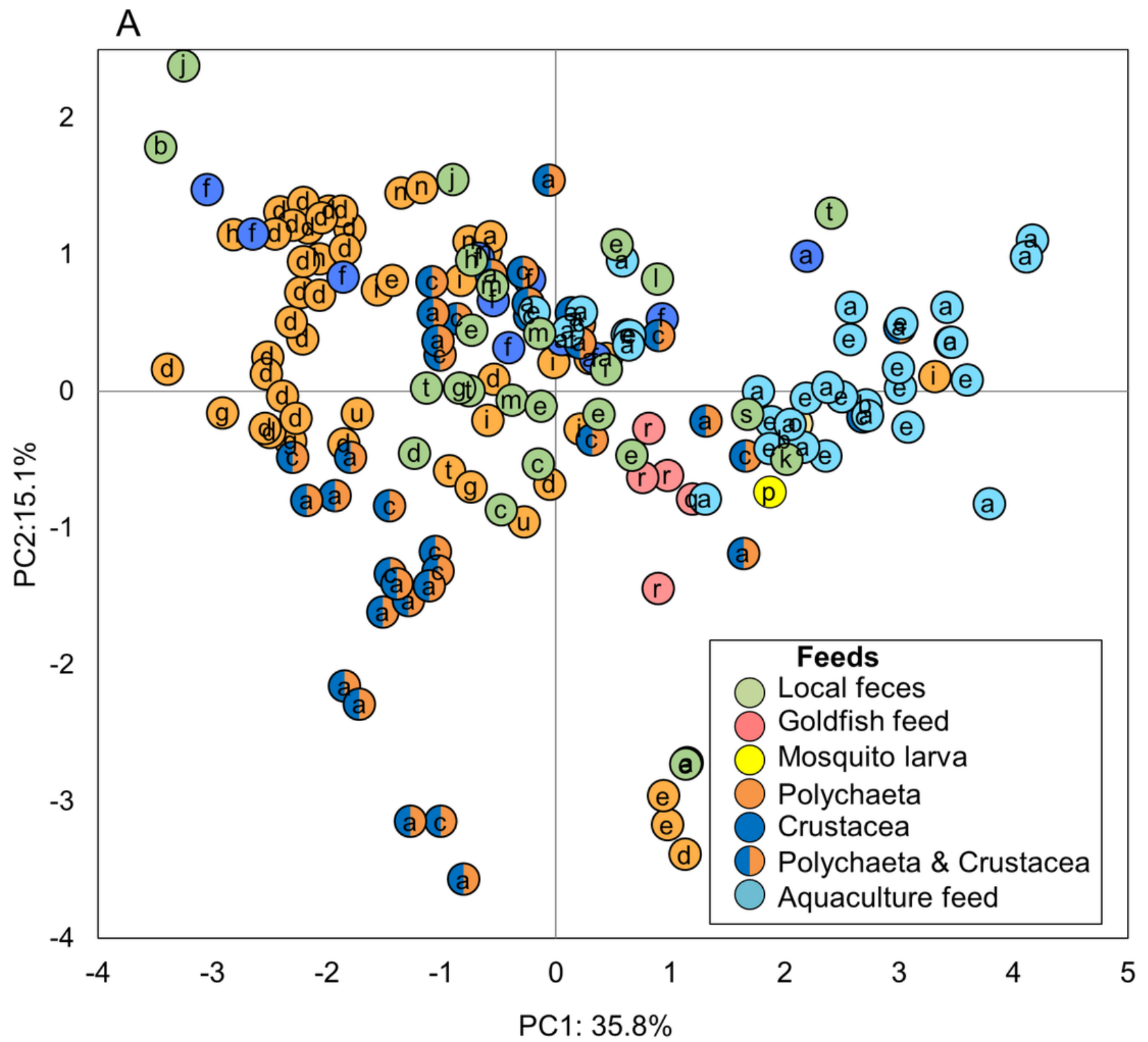

B

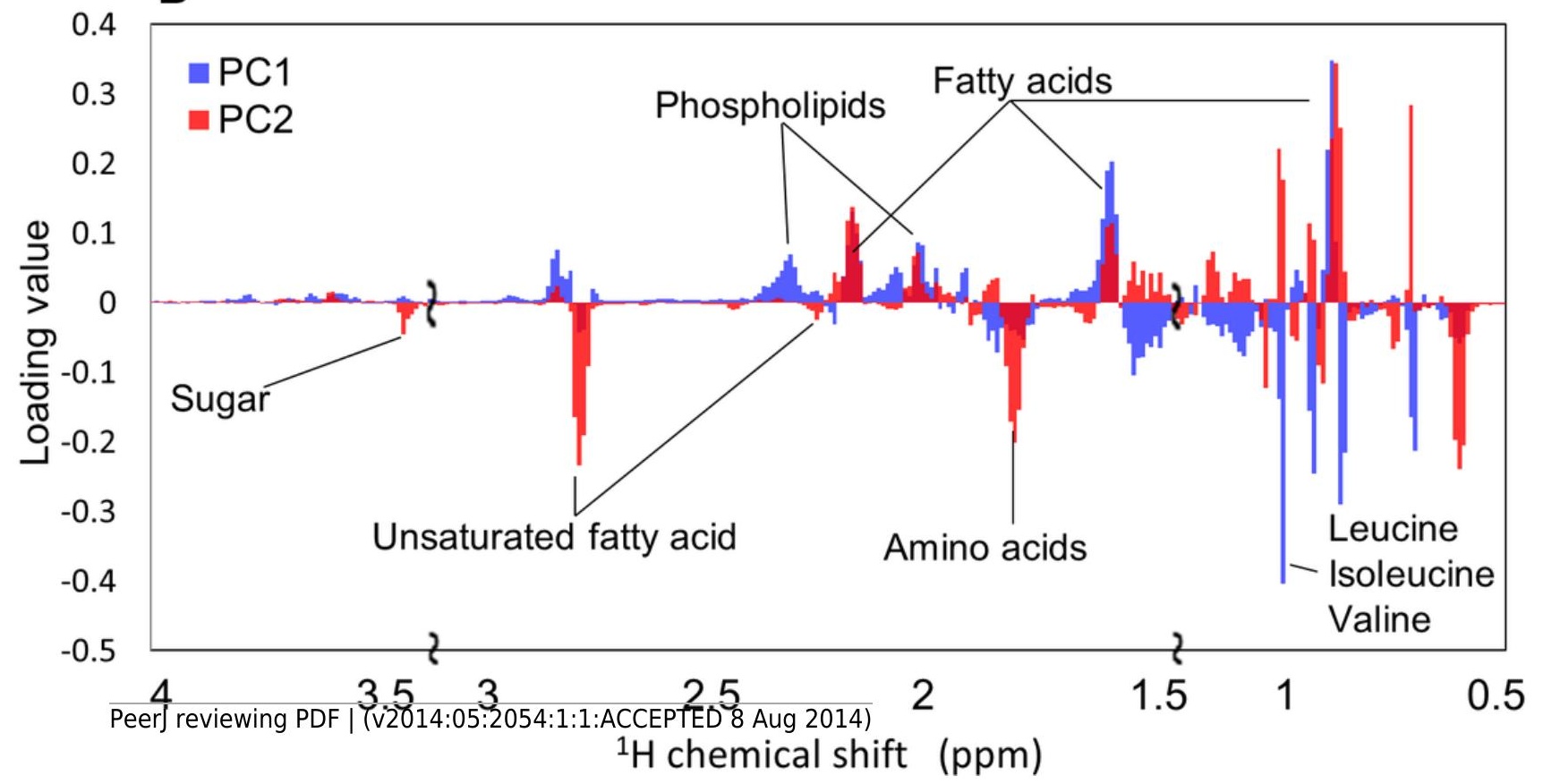




\section{Figure 6}

Figure 6.

Metabolic and microbial profiles of feeding response in Epinephelus septemfasciatus feces.

Symbols representing individual communities are colored by diet (red: carnivorous feeding, green: aquaculture feeding). Three Epinephelus septemfasciatus were bred by carnivorous feeding for 3 months. They were then divided into three tanks $(*,+, \neq)$, and bred for 3 weeks by aquaculture feeding. One E. septemfasciatus was then bred with aquaculture feeding, and the others were bred with carnivorous feeding. (A) PCA score plot on ${ }^{1} \mathrm{H}$ NMR profile from feces $\left(n=38, k=808, R^{2} X=0.488, R^{2} Y=0.187, Q^{2}=0.442\right)$. (B) PCA loading plots on ${ }^{1} H$ NMR profiles. Letters $S, U, P, A, F, L, I$, and $V$ indicate sugar, unsaturated fatty acid, phospholipids, amino acids, fatty acids, leucine, isoleucine, and valine, respectively. (C) Bacterial profile that changed characteristically during the 4 months of rearing. These data were first normalized by total reads for each sample (i.e., the ratio in each fecal sample), and then secondarily normalized on the basis of the sum of the ratios of the same bacteria. 


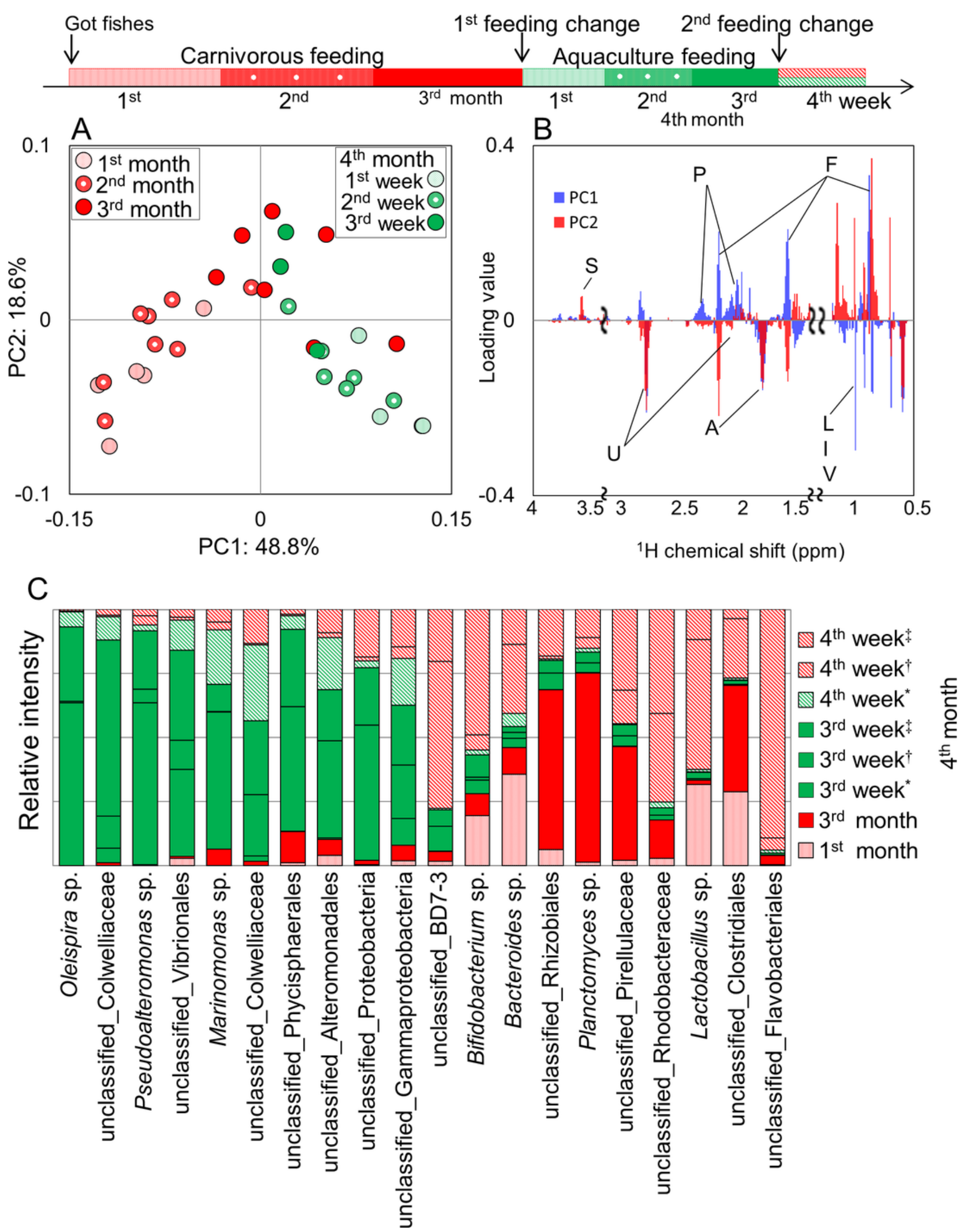

